have been met. The small percentage of "medical" withdrawals $(14 \%)$ suggests a satisfactory level of acceptability.

The low-oestrogen combined oral contraceptive can therefore be recommended for general use.

We gratefully acknowledge the help of the many doctors who contributed cases to this trial. We also acknowledge the participation of the Medical and Scientific Information Departments of John Wyeth \& Brother Ltd. in planning part of the trial.

\section{References}

Adelstein, A. M. (1972). Health Trends, 4, 2

Goldzieher, J. W., Moses, L. E., Averkin, E., Scheel, C., and Taber, B. Z. (1971). Fertility and Sterility, 22, 609.

Greenblatt, R. B., Jungck, E. C., Puebla, R. A., and Ward, M. C. (1966). Clinical Pharmacology and Therapeutics, 7, 490.

Hallberg, L., Hogdahl, A. M., Nilsson, L., and Rybo, G. (1966). Acto Obstetricia et Gynecologica Scandinavica, 45, 320.

Meyler, L., and Herxheimer, A. (1966). Side-effects of Drugs, 5, 530.

Potter, R. G. (1966). Demography, 3, 297.

\title{
Value of Cuff Occlusion Pressures in Assessment of Peripheral Vascular Disease
}

\author{
C. L. CUTAJAR, ADRIAN MARSTON, J. F. NEWCOMBE
}

British Medical fournal, 1973, 2, 392-395

\section{Summary}

Cuff occlusion pressures using the ultrasonic Doppler machine were recorded in 30 normal legs and in 90 legs with peripheral vascular disease. With this technique it was possible to show obstructions to the circulation above and below the inguinal ligament.

\section{Introduction}

The diagnosis and management of peripheral arterial disease depends on clinical and arteriographic findings. There is increasing awareness of the fact that arterial occlusions as seen on an aortogram may not always correspond with the severity of the patient's symptoms. Stenotic lesions, which seem unimportant on the $x$-ray picture, may in fact be responsible for a significant pressure drop and diminished blood flow. The introduction of the ultrasonic blood-flow velocity detector has made it possible to detect arteries which, while patent, are impalpable, because of proximal arterial occlusion, ankle swelling, or obesity.

In the present study this instrument has been used to record cuff occlusion pressures in the lower limbs of normal subjects and of patients with peripheral vascular disease.

\section{Technique}

The transcutaneous blood velocity detectors used in this study were the Doppler 801 and 802 developed by Parks Laboratories (Beaverton, Oregon, U.S.A.). The physical principles underlying the use of this instrument have been described by Franklin et al. (1961), Strandness et al. (1965), and Rushmer et al. (1966). A Devices two-channel recorder (model M2) was used to record the wave trace.

With the patient lying, cuffs are placed around the thigh, calf, and ankle. For the calf and ankle measurements standard sphygmomanometer cuffs are used, one being placed above the malleoli and the other below the knee. For the thigh a special

Department of Surgical Studies, The Middlesex Hospital, London W1 C. L. CUTAJAR, M.D., F.R.C.S., Commonwealth Research Scholar (Present address: 49 Amery Street, Sliema, Malta)

ADRIAN MARSTON, D.M., M.CH., Consultant Surgeon

Department

J. F. NEWCOMBE, M.CHIR., F.R.C.s., Consultant Surgeon cuff $68-\mathrm{cm}$ long (Electro-Medical Engineering, California) is used, the inflatable portion measuring 10 by $40 \mathrm{~cm}$. It is essential for this cuff to go comfortably around the limb with an adequate overlap. An aneroid manometer recording pressure in $\mathrm{mm} \mathbf{~ H g}$ is attached.

The brachial occlusion pressure is first recorded with the Doppler probe placed over the artery at the elbow. The pressure is read in both arms and the highest reading noted. In practice the difference in pressure between the two arms is seldom more than $5-10 \mathrm{~mm} \mathrm{Hg}$. If both legs are being studied it is advisable to record the brachial pressures again before the second limb is investigated. The most audible pedal pulse is then selected and the pencil-probe placed at this site at an angle of $45^{\circ}$. The thigh cuff is inflated above systolic pressure and then gradually let down, $5 \mathrm{~mm}$ at a time, until an arterial signal is again heard. This is taken to denote the thigh occlusion pressure. The same procedure is repeated with the calf and ankle cuffs, keeping the ultrasound probe directed to the same spot overlying a pedal artery.

When the test is performed after operation, care should be taken not to place the cuff over a tender area. This problem is most commonly met in the thigh after a femoropopliteal reconstruction. In these circumstances it is necessary to postpone the thigh readings until the tenderness subsides.

In this study we have expressed the cuff occlusion pressures at the thigh, calf, and ankle as a percentage of the brachial pressure. This makes it possible to compare not only pressure in different patients but also in the same subject when the pressures are taken on different occasions.

\section{Patients Studied}

Sixty patients with intermittent claudication aged from 37 to 69 years of age were studied (see table). Two patients underwent femoral arteriography, the remainder all had a translumbar aortogram. Ninety legs were found to have arterial occlusions, of which 45 were confined to the superficial femoral artery. The remaining 45 legs had an aortoiliac block, 25 of which had an associated block in the femoral artery.

Twenty-six patients undergoing vascular surgery were studied before and after operation.

Nature of Vascular Disease in Subjects Studied

\begin{tabular}{|c|c|c|}
\hline & $\begin{array}{l}\text { No. of Subjects } \\
\text { Studied }\end{array}$ & $\begin{array}{l}\text { No. of Legs } \\
\text { Affected }\end{array}$ \\
\hline 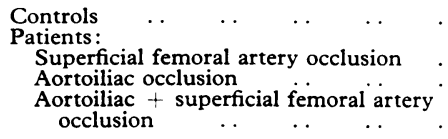 & $\begin{array}{l}30 \\
30 \\
10 \\
20\end{array}$ & $\begin{array}{l}30 \\
45 \\
20 \\
25\end{array}$ \\
\hline
\end{tabular}


Thirty patients ranging from 21 to 60 years of age with full pulses and no history of coronary or cerebral disease were also studied. This group served as controls.

\section{Results}

\section{THIGH OCCLUSION PRESSURES}

The thigh cuff occlusion pressures (expressed as a percentage of the brachial) in subjects with and without peripheral vascular disease are shown in fig. 1 .

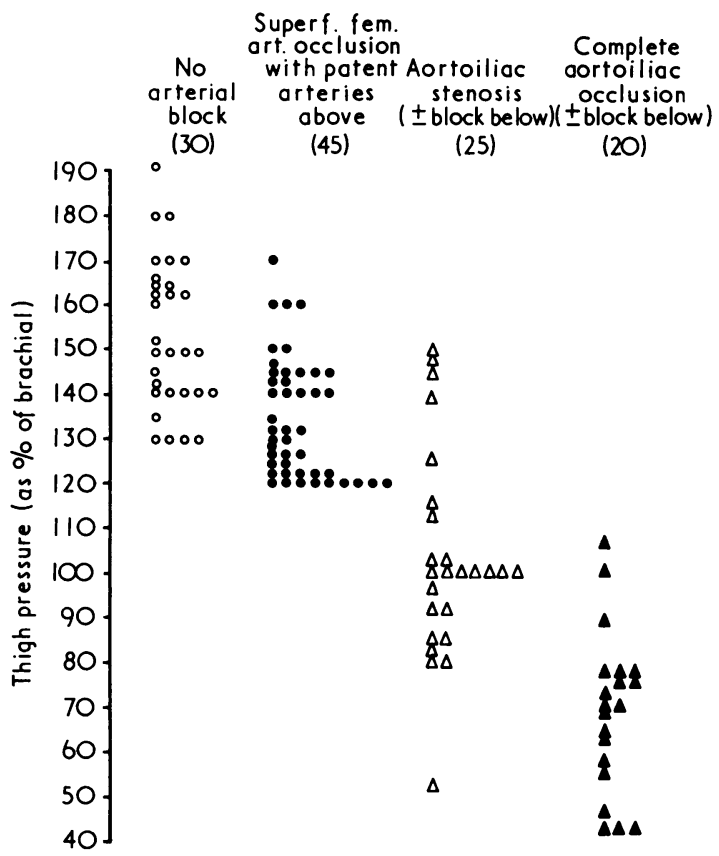

FIG. 1-Thigh cuff occlusion pressures in patients with and without vascular disease.

In the control group thigh occlusion pressures ranged from $130 \%$ to $190 \%$ (mean $153.3 \%$, S.D. 16.6). In absolute terms the smallest thigh/brachial difference observed was $30 \mathrm{~mm} \mathrm{Hg}$ and the greatest $120 \mathrm{~mm} \mathrm{Hg}$. This difference between the recorded thigh and brachial pressures is due to the relatively large volume of the thigh interposed between cuff and artery. In normal supine subjects there is no significant difference in the pressures in the brachial and femoral arteries if measurements are made by an intra-arterial technique.

The legs with a superficial femoral artery occlusion but with no radiological evidence of an aortoiliac block showed thigh occlusion pressures relating to the brachial, somewhat lower than the control group, with a range of $120 \%$ to $170 \%$ (mean $134.2 \%$, S.D. 13.4). Although this difference from the controls is statistically significant $(t=5.47, \mathrm{P}<0.001)$, it is noteworthy that in simple superficial femoral blockage the thigh cuff occlusion pressure remains greater than the brachial.

By contrast, in the group with a complete aortoiliac block the thigh occlusion pressures were, with only one exception, lower than the brachial, and fig. 1 shows that 17 of these 20 legs had a thigh pressure of less than $80 \%$ of the brachial. The mean systolic pressure was $68.8^{\circ} \%$ of the brachial, which was significantly less than that obtained either in the control group $(t=16.8, \mathrm{P} 0.001)$ or in legs with radiological evidence of superficial femoral artery occlusion $(t=16 \cdot 11, \mathrm{P} 0.001)$.

In the group of 25 legs in which the aortoiliac segment was narrowed but not completely blocked, all but one case showed a thigh pressure greater than $80 \%$ of the brachial with a mean of $103.2 \%$ (S.D. 23.4). Four cases with radiological evidence of stenosis were found to have thigh occlusion pressures greater than $130 \%$ of the brachial-that is, within the control range.
These results indicate that measurement of the thigh/ brachial pressure ratio enables patients broadly to be classified as follows:

(1) $120 \%$ or greater . . No significant aortoiliac occlusion.

(2) $80 \%-120 \% \quad$. $\quad$ Significant aortoiliac stenosis but probably not complete occlusion.

(3) Less than $80 \% \quad$. . Probably complete iliac occlusion.

\section{CALF AND ANKLE OCCLUSION PRESSURES}

The calf and ankle cuff occlusion pressures in the control group and in patients with intermittent claudication are shown in figs. 2 and 3.

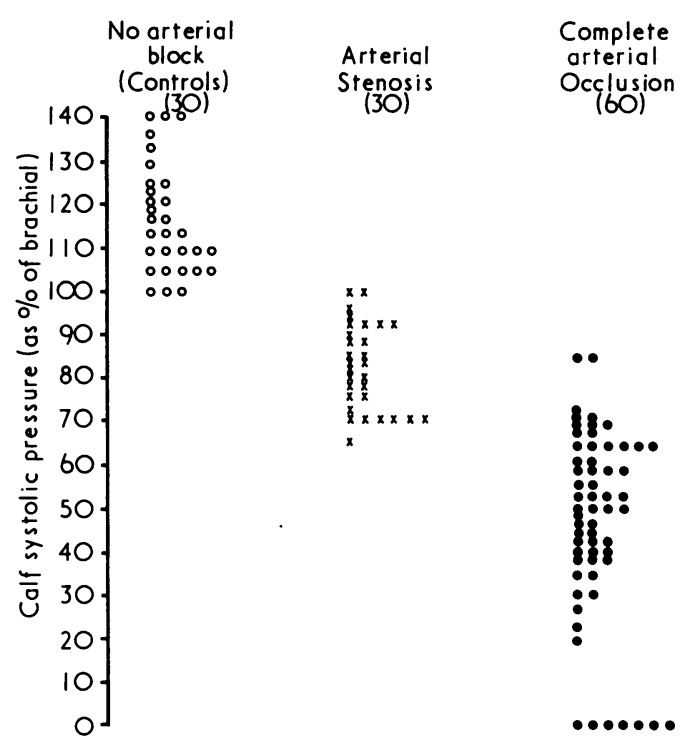

FIG. 2-Calf cuff occlusion pressures in patients with and without vascular disease.

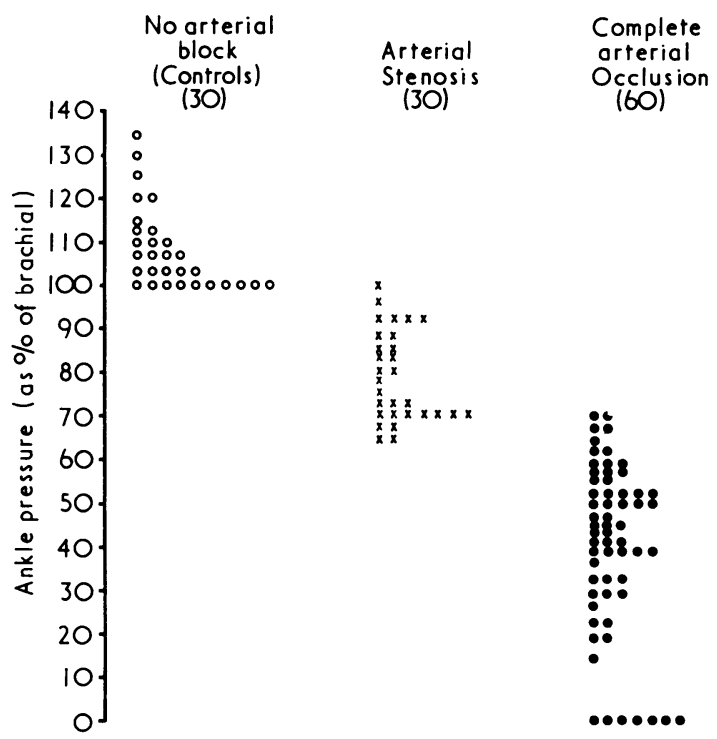

FIG. 3-Ankle cuff occlusion pressures in patients with and without vascular disease.

The legs in the control group all had calf occlusion pressure which ranged between $100^{\circ} \%$ and $140 \%$ of the brachial level (mean $116.5 \%$, S.D. 12.5). The ankle occlusion pressure had a very similar range $(100 \%$ to $135 \%$; mean $108.3 \%$, S.D. 9.4).

The patients with an arterial block were divided into two groups depending on the radiological appearance. The group 
of 30 legs with an incomplete arterial block above the level of the popliteal trifurcation had a calf pressure range between $65 \%$ to $100 \%$ of brachial (mean $82.4 \%$, S.D. $10 \cdot 2$ ). In this group all except one leg had a pressure of $70 \%$ or over. The corresponding ankle occlusion pressure had a similar range (65\% to $100 \%$, mean $78 \cdot 8 \%$, S.D. $10 \cdot 1$ ).

Sixty legs showed a complete arterial occlusion at one or more sites above the level of the popliteal trifurcation. In seven the pressure could not be determined as no pedal pulse was heard. The remaining 53 had calf pressures ranging between $20 \%$ and $85 \%$ (mean $45.9 \%$, S.D. 21.6 ). Only five of these had pressures of $70 \%$ or over. The corresponding ankle pressures (excluding the legs without audible pulses) had a range of $15 \%$ to $70 \%$ (mean $40 \cdot 76 \%$, S.D. $19 \cdot 7$ ).

Considering the whole group of 90 legs with intermittent claudication, the following two generalizations can be made.

(1) In contrast to the control group, calf and ankle pressures are never more than brachial level.

(2) A calf systolic pressure lower than $70 \%$ or an ankle pressure lower than $60 \%$ of the brachial level represents a complete arterial occlusion. The block may be anywhere from the aorta to the termination of the popliteal artery. Measurement of the thigh occlusion pressure will give more information on the probable site of the block, but accurate identification will depend on arteriography.

The calf-ankle gradient in patients with peripheral vascular disease showed no significant difference from that of the control group as shown in fig. 4. The mean calf-ankle pressure gradient

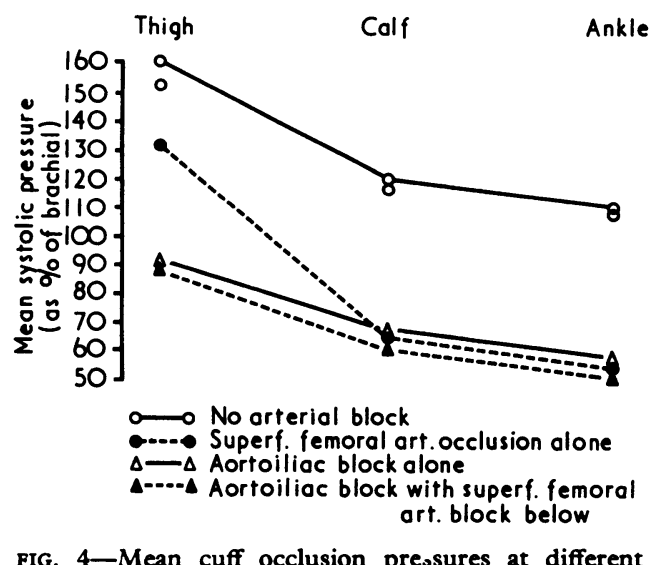

FI. 4-Mean cuff occlusion pressures at different

(expressed as a percentage of the brachial) was $8.6 \%$ compared with $8 \%$ in the control group. In practice, therefore, to obtain a distal cuff occlusion pressure, one need record this only at the ankle. This is more convenient and accessible than the calf, especially after reconstructive vascular surgery.

An attempt was made to correlate the patients' symptoms with ankle occlusion pressures (fig. 5). There were 23 legs

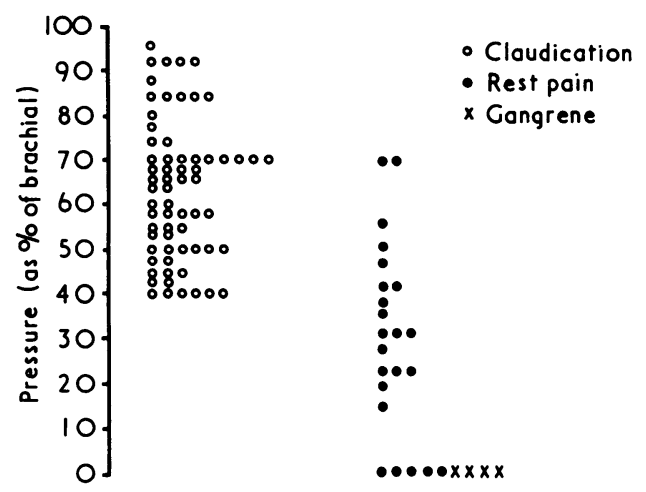

FIG. 5-Relation between ankle cuff occlusion pressure and symptoms in legs with peripheral vascular disease. investigated for rest pain and the mean ankle pressure in this group was $28 \%$ of the brachial.

Patients with intermittent claudication but with no rest pain had relatively higher pressures, all 65 legs in this group having a pressure of $40 \%$ or more, with a mean of $63 \cdot 2 \%$.

It therefore appears that there is a correlation between ankle occlusion pressures and symptoms as follows: (a) an ankle occlusion pressure of less than $40 \%$ of the brachial indicates severe ischaemia and is commonly associated with rest pain; (b) an ankle occlusion pressure of more than $40 \%$ is rarely associated with rest pain.

\section{CUFF OCCLUSION PRESSURES BEFORE AND AFTER VASCULAR SURGERY}

The study of occlusion pressures before and after arterial reconstruction may be used in the assessment of the results of vascular surgery.

Calf and ankle pressures are shown in fig. 6 which were measured one to two weeks after operation in 16 patients who were successfully treated for an isolated arterial occlusion. Two of these patients underwent arterial surgery above the inguinal ligament (aortoiliac disobliteration or bypass graft) and the remaining six had a femoral-popliteal bypass. All showed a significant increase in the postoperative calf and ankle pressure readings. In all cases the calf pressure rose to the normal range-that is, it was equal to or higher than the brachial. The ankle pressure, however, was converted to "normal" in only six of the 16 patients, the remaining 10 patients having an ankle pressure of between $90 \%$ and $95 \%$ of the brachial.

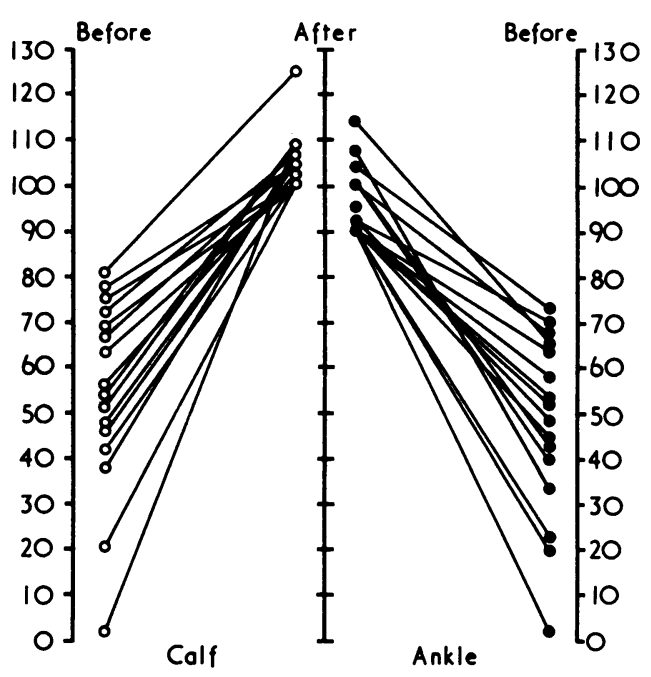

FIG. 6-Calf and ankle pressures in 16 patients before and after vascular surgery.

It would appear, therefore, that reconstructive arterial surgery can be regarded as having been successful if the postoperative calf pressure is $100 \%$ of the brachial or more and the ankle pressure $90 \%$ or more.

A similar improvement in thigh pressure was noted in patients undergoing successful vascular surgery above the inguinal ligament. The results in 14 of these patients are shown in fig. 7. It should be noted that despite complete symptomatic improvement six of these patients had relatively low (less than $130 \%$ ) postoperative thigh pressures. Four of these, however, had a residual superficial femoral artery occlusion, and as has been noted above, this has the effect of reducing the thigh occlusion pressure.

Ten patients who underwent unsuccessful vascular surgery for an isolated arterial block had low postoperative calf and 


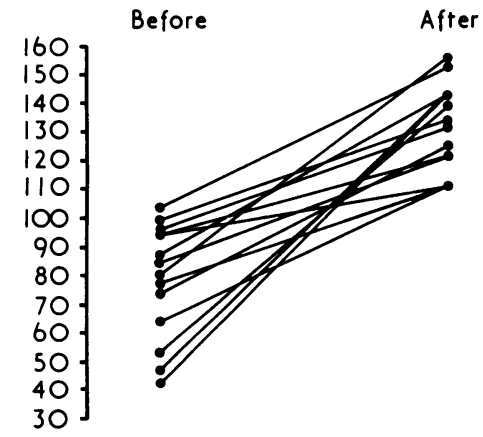

FIG. 7-Thigh pressures before and after vascular surgery above inguinal ligament in 14 patients.

ankle pressures. In all cases the reconstructed artery or graft occluded within a week after surgery. In only one of these patients was the ankle pressure appreciably improved (from $50 \%$ to $62 \%$ ) with slight symptomatic improvement. In five patients the ankle pressures before and after operation remained virtually the same with no significant change in the patient's symptoms. One of these patients, with inaudible pedal pulses both before and after surgery, eventually had an above-knee amputation. In the remaining four patients there was a deterioration in ankle pressures with unchanged or worse symptoms after operation; one patient in this group had to have an aboveknee amputation.

One important fact which emerges from this series is that a low preoperative peripheral pressure is not in itself a contraindication to major vascular surgery. The mean preoperative ankle systolic pressure in the "failed" group was $32 \%$ compared with $59 \%$ in the "success" group. Three patients in the latter group, however, had preoperative ankle pressures of less than $20 \%$ of brachial. All three did very well after bypass graft operations, the ankle pressure rising to $90 \%$ and the graft remaining patent. Two of these patients had a good run-off on $x$-ray examination, but one of them had only one fully patent artery in the leg. On the other hand a relatively high preoperative ankle pressure is not a guarantee to a good operative result, three of the "failed" group having preoperative ankle pressures between $50 \%$ and $60 \%$ with a reasonably good runoff. When considering the series as a whole it seems, nevertheless, that a successful outcome to reconstructive vascular surgery is to be expected more confidently with a preoperative ankle pressure of $50 \%$ or over.

\section{Discussion}

In the preoperative assessment of patients with peripheral vascular disease, arteriography is undoubtedly the most important ancillary investigation. Unfortunately, with the conven- tional single-plane arteriogram it is often difficult to interpret the functional significance of an occlusion, especially if this is incomplete. An apparently minor stenosis seen on the $x$-ray picture may, in fact, have a significant pressure gradient across it. Conversely, when the collateral circulation is well developed, a complete block may cause a fall in pressure which is very much less than would be expected. In recent years the development and use of such techniques as mercury strain gauge plethysmography (Strandness and Bell, 1965), capacitance pulse pickups (Carter, 1968), and the transcutaneous ultrasound (Doppler) flowmeter (Strandness and Bell, 1966, Yao et al., 1968) have made it possible for the clinician to study occlusion pressures in the absence of palpable distal pulses. In our hands, the transcutaneous Doppler device has proved to be a simple, reliable apparatus for this purpose.

The results of the present study indicate the following.

(1) In a normal limb the calf and ankle occlusion pressure is equal to or higher than the brachial. If the pressure at any of these sites is lower than the brachial then there is an occlusion either in the aortoiliac or femoropopliteal system.

(2) In patients with peripheral vascular disease a thigh pressure which is higher than the brachial by at least $20 \%$ indicates that there is no significant aortoiliac lesion.

(3) With a thigh pressure greater than the brachial and a calf or ankle pressure less than the brachial, an isolated superficial femoral artery occlusion can be diagnosed. In these circumstances the ankle pressure correlates well with the severity of the patient's symptoms.

(4) After successful arterial reconstruction there is always an increase in pressure at the calf and ankle over the preoperative level. The calf pressure should be equal to or greater than the brachial, and ankle pressure should rise to at least $90 \%$ of that level. After successful aortoiliac surgery there is always a substantial increase in thigh pressure, usually to a level above $120 \%$ of the brachial.

Our thanks are due to Mr. G. Turney, Mr. H. S. Drury, and Miss $\mathrm{K}$. Wong, of the Department of Medical Illustration, The Middlesex Hospital Medical School. The pressure measuring equipment was purchased by means of a generous grant from the Central Research Fund, University of London.

\section{References}

Carter, S. A. (1968), Circulation, 37, 624.

Franklin, D. L., Schlegel, W. A., and Rushmer, R. F. (1961). Science, 134, 564.

Rushmer, R. F., Baker, D. W., and Stegall, M. F. (1966). Fournal of Applied Physiology, 21, 554.

Strandness, D. E., and Bell, J. W. (1965). Annals of Surgery, Suppl. No. 4,

Yao, S. T., Hobbs, J. T., and Irvine, W. T. (1968). British Medical fournal, 4,555 . 